\title{
The Research of Modeling Method of Railway 3D Landscape
}

\author{
LV Xikui ${ }^{1,2}$, Wang Mingsheng ${ }^{3}$ and $\mathrm{He} \mathrm{Bin}^{3}$
}

${ }^{I}$ School of Traffic and Transportation, Shijiazhuang TieDao University, Shijiazhuang, ${ }^{2}$ Traffic Safety and Control Lab of Hebei Province, Shijiazhuang, China ${ }^{3}$ School of Civil Engineering, Shijiazhuang TieDao University, Shijiazhuang, China swedenass@126.com ${ }^{1,2}$,wms@stdu.edu.cn ${ }^{3}$,hebin@stdu.edu.cnl ${ }^{3}$

\begin{abstract}
It's very important for environment simulation in the three dimensional railway line selection and design, and the establishment of three-dimensional landscape model impacts the fidelity of simulation environment directly. The paper studied. Of the $3 D$ landscape modeling methods and key technologies. The paper achieved three-dimensional modeling of railway line used bi-cubic coons model, realized line structures three-dimensional modeling by object-oriented modeling approach. Based on Visual C -5 and Oracle databases, The paper established the structure model library and realized the effective management of the model, Solved the exact match and control problem between structure mode and the threedimensional scene, Achieved the application of structure mode in three-dimensional line landscape simulation and established a three-dimensional line landscape with higher fidelity. Developed a three-dimensional landscape line annamic environment simulation system based on the methods and theories of paper studied. Using this system, Yunnan - Tibet railway line has been designed 3D landscape modeling, Verified the correctness of the theory and algorithms of paper studied.
\end{abstract}

Keywords: railyay line; 3D landscape; landscape model; modeling; model library.

\section{Introduction}

Three-dimensional vsualization of the railway line design has an important role in investigate the design and improve the design quality; the establishment of three-dimensional landscape model is the key to achieving design and impact the fidelity of simulation environment directly. The railway line 3D landscape modeling including lines, bridges, structures and other ancillary facilities Three-dimensional modeling. Through virtual reality the reality of line landscape model, Allows designers to see their various designs intuitively, Assisted analysis, evaluation, planning or decision-making, and then improved the design speed and design quality. Therefore, it has important theoretical and practical value to study the modeling method of railway line 3D landscape.

\section{Three-dimensional modeling of design line}

The design line is a major component of the railway, it include road sleepers, ballast, ballast slope, shoulder, side ditch, slope protection Slope protection, etc. They are express through triangulation surface object of several polygon boundaries. The data of 
generating the design line from virtual environment railway location system, the feature point data of line can be expressed as following structure:

Struct SectionPoint

\{ double $\mathrm{x}, \mathrm{y}, \mathrm{z}$; // Record the three-dimensional spatial coordinates of feature points int pointType; // the type of feature point, as shown in Table 1 and Figure 1.

Table 1. Type code of feature points

\begin{tabular}{|c|l|c|l|}
\hline Type Number & Category Description & Type Number & Category Description \\
\hline 0 & Line Center & 6 & ballast \\
\hline 1 & side ditch & 7 & sleeper \\
\hline 2 & side ditch Platform & 8 & rail \\
\hline 3 & subgrade Platform & 9 & slope platform \\
\hline 4 & shoulder & 10 & ground line of slope \\
\hline 5 & ballast Platform & & \\
\hline
\end{tabular}

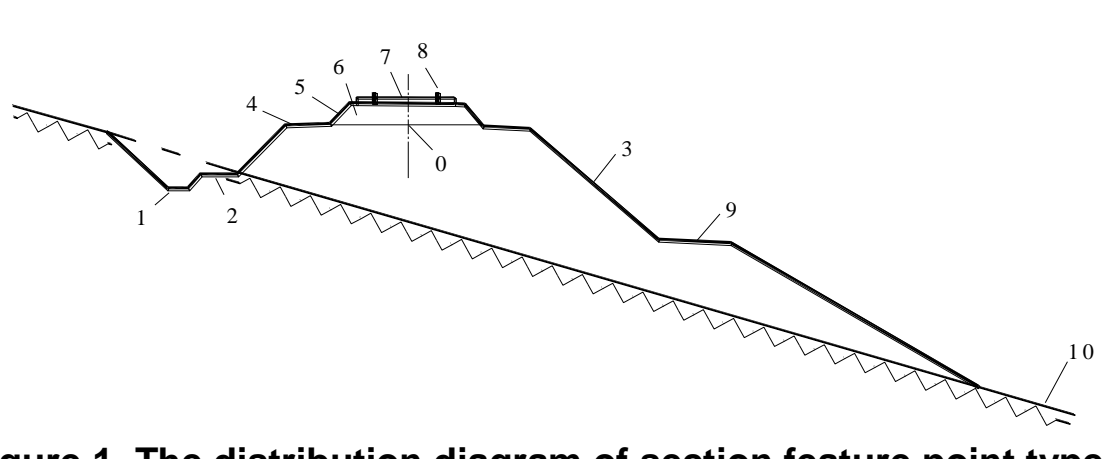

Figure 1. The distribution diagram of section feature point type

Coons surface model was a two-parametric surface model, Expressed in vector form as $P(u, v)=[x(u, v), y(u, v), f(v), v)$, It consists of four boundary curves $p(u, 0), \quad p(u, 1)$, $p(0, v), \quad p(1, v)$. The Corner, border and cross-cut vector of coons patch was shown in figure 2[1].Coons surfaces is suitable for expressing design lines surface, It has been applied in line surface mode [H] and at-grade intersections design[2,3].

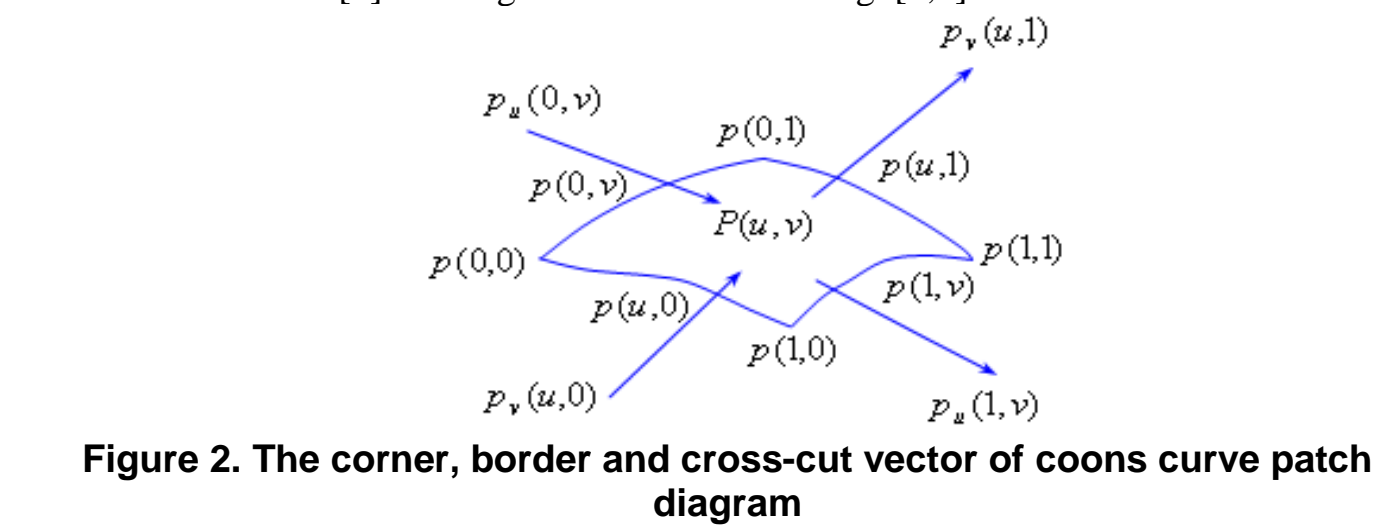

In the Coons curve model, the bi-cubic coons surface patches adopt Cubic Hermite interpolation function and achieved $\mathrm{C} 1$ smooth connection [4]. Therefore, the paper chose bicubic Coons surface as description model of line design surface. Built bi-cubic Coons patch structure as follows. 
(1)According to the boundary curve $p(0, v), p(1, v)$ and cross-cut vector $p_{u}(0, v)$ and $p_{u}(1, v)$, built surface patches $p_{1}(u, v)$ along the parameter $u$ direction, as shown in formula 1 .

$$
P_{1}(u, v)=\left[\begin{array}{llll}
F_{0}(u) & F_{1}(u) & G_{0}(u) & G_{1}(u)
\end{array}\right]\left[\begin{array}{c}
P(0, v) \\
P(1, v) \\
P_{u}(0, v) \\
P_{u}(1, v)
\end{array}\right]
$$

In formula $1, F_{0}(u), \quad F_{1}(u), \quad G_{0}(u), \quad G_{1}(u)$ were cubic Hermite basis functions.

$$
\left\{\begin{array} { l l } 
{ F _ { 0 } ( u ) = 2 u ^ { 3 } - 3 u ^ { 2 } + 1 } \\
{ F _ { 1 } ( u ) = - u ^ { 3 } + 3 u ^ { 2 } }
\end{array} \left\{\begin{array}{l}
G_{0}(u)=u(u-1)^{2} \\
G_{1}(u)=u^{2}(u-1)
\end{array}\right.\right.
$$

(2)According to the boundary curve $p(u, 0), p(u, 1)$ and cross-cut vector $p_{v}(u, 0)$ and $p_{v}(u, 1)$, built surface patches $p_{2}(u, v)$ along the parameter direction, as shown in formula 2.

(3)Construct bi-cubic coons surface patches $P(u, N)$

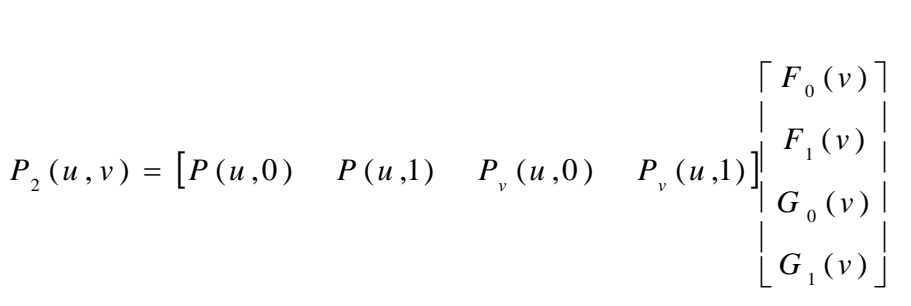

The simply Superposition between $P_{1}(u, \hat{v})$ and $P_{2}(u, v)$ can't get required patches due to the repeated application of the four coners point's vector, tangent vector and mixed derivatives. Therefore, it mast subtract some extra items $P_{3}(u, v)$ from $P_{1}(u, v)+P_{2}(u, v)$ in order to get the bi-cubic coons surface patches

$$
\begin{array}{ccccc}
P(u, v) & =P_{1}(u, v)+P_{2}(u, v)-P_{3}(u, v), \quad u, v \in[0,1]
\end{array}
$$

In formula 4 , it need provide four cross-border tangent vectors and four corners points twist vector except four boundary curves. The four cross-border tangent vector were reasonable to use Adini was shown in formula 5.

$$
P_{u v}(u, v)=P_{v u}(u, v)=-P_{v}(0, v)+P_{v}(1, v)-P_{u}(u, 0)+P_{u}(u, 1)+c
$$

In formula $5, c=P(0,0)-P(0,1)-P(1,0)+P(1,1)$, It can get four corners points twisting vector through substituted $u, v=0,1$ 1into formula 5 respectively.

(4) Realization of design line coons' surface model 
By calculating the coordinates of the corner point of line's route section, The corresponding corner of adjacent cross section connected together to form a curvilinear quadrilateral mesh [5]. The entire design line surface composed by these grids and each grid consists of bi-cubic coons patches, as shown in Figure 3, Figure 4 is a grid cell from Figure 3.

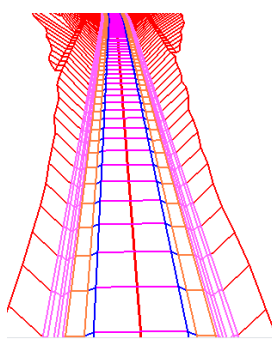

Figure 3. The line 3D surface model

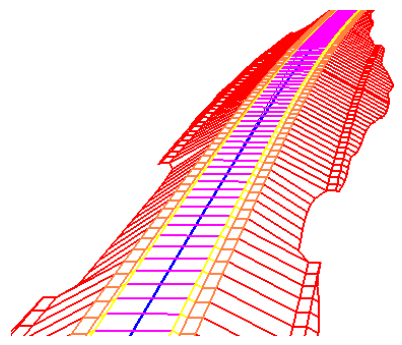

In Figure 4:

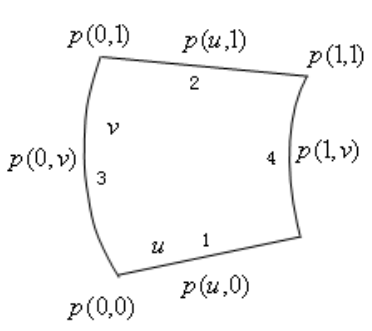

Figure 4. The bi-cubic coons surface patches

(1)The edge 1 and edge 2 corresponding to the normal direction of the line, Plane and elevation were straight lines.

(2)The edge 3 and edge 4 corresponding to the line forward direction, The plane can be linear, circular or transition curve, Facade can be linear, circular or other space curves.

It can list the four boundary curve equation based on the analysis results, and then calculated the four corners of Adini twist vector and the four cross-border tangent according to the farin method. Finally, constructed bi-cubic coons patches according to equation 5, the modeling results of three-dimensionalline shown in Figure 5.

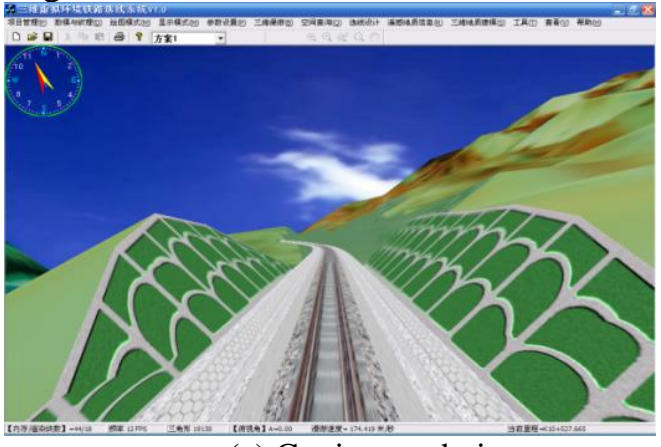

(a) Cutting renderings

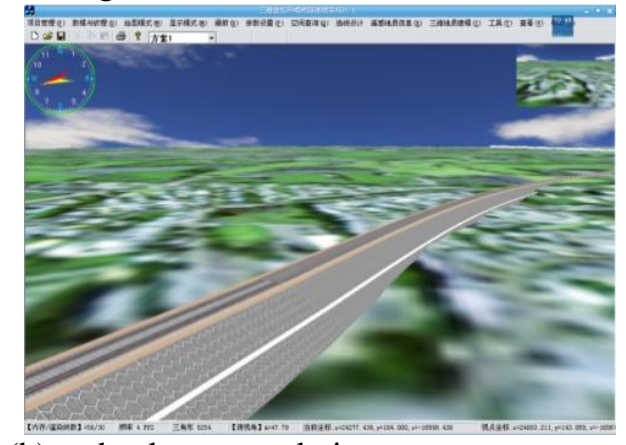

(b) embankment renderings

Figure 5. The line three-dimensional modeling renderings

\section{Three-dimensional modeling of the line structures}

\subsection{Object-oriented graphics assisted simulation modeling}

The basis of object-oriented graphical modeling is the system divided, namely the system is considered by the subsystems, and subsystems can be decomposed into more primitive subsystem [6]. Because of the presence of this nature, the method of building the overall model by connecting the sub-model, this related to the model-level division. According to the solid modeling ideas based on object-oriented and different types of railway line structures, functions and attributes, Division principles as follows:

(1)Divided into units as a separate object model; 
(2)Unit model can be spliced into the overall model easily;

(3) The same type of model units can be interchanged to create a new global model.

The bridge as an example to illustrate:

Pier + supported beam + Bridge Deck $=$ Bridge

Namely, the pier, supported beam and bridge deck as basic element model for the bridge division and can't broke down, shown in Figure 6. Transformed some units model can implement new 3D landscape, shown in Figure 7 and Figure 8.

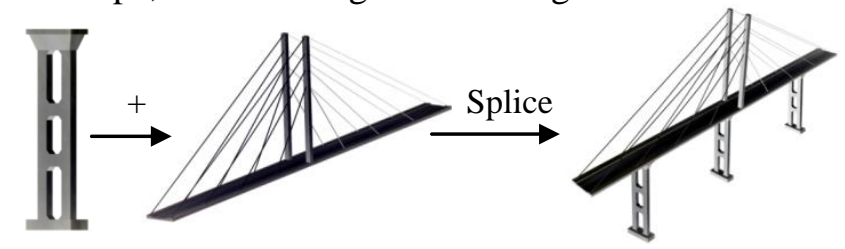

Unit model Unit model Overall model

Figure 6. The schematic diagram of overall model general ed by unit model

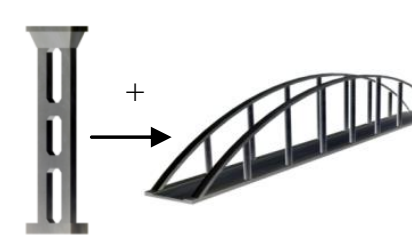

Figure 7. Generate new overal models by converting part of cell model

Figure 7. Generate new overallmodels by converting part of cell model

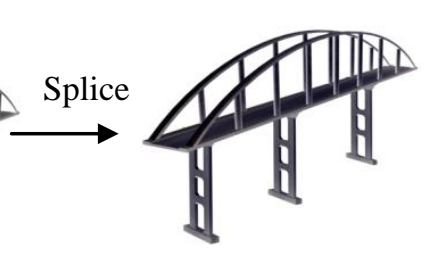

Oyerall model

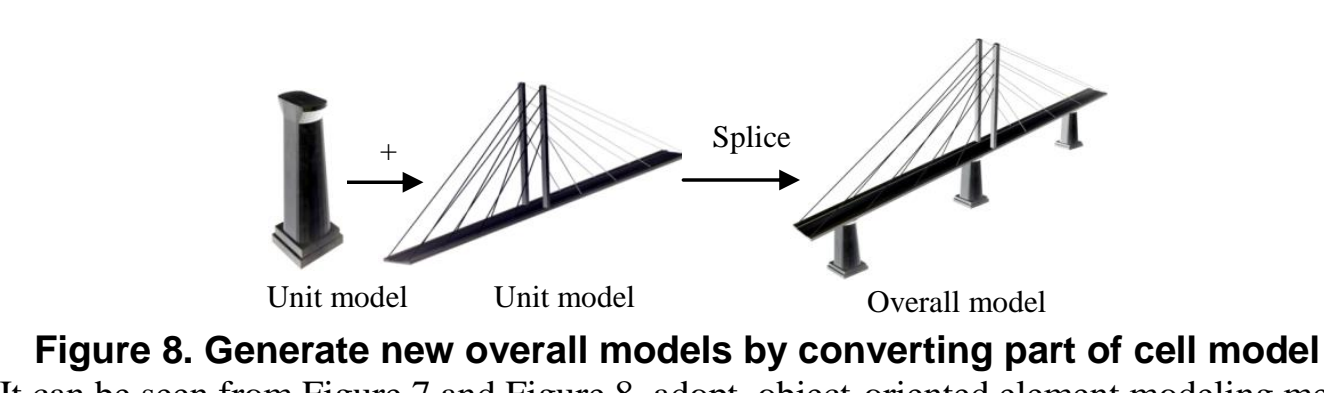

It can be seen from Figure 7 and Figure 8, adopt object-oriented element modeling method, The new whole model ean be generated by combining the element model, and can reduce the modeling workload an Onodel number effectively. The mathematical logic expression was shown below:

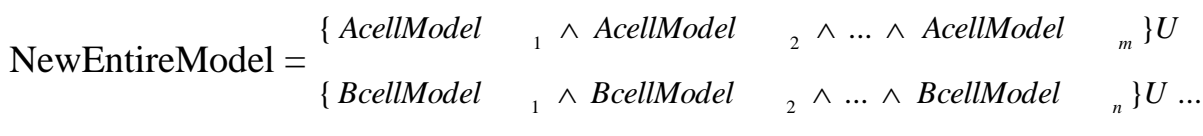

\subsection{Three-dimensional model library of Structures}

in order to y manage the model, reuse and accelerate modeling more effectively, It's a better solution to management model, model elements and texture data by database.

(1) The structure of the database table

The corresponding texture images of various 3D models were independent of the model, Therefore, models and textures data were stored in binary fields (BLOB). Meanwhile, in order to improve the efficiency of management, query and retrieval, also designed the model type and model name field, the table structure shown in Table 2. 
Table 2. The table structure of 3D model database

\begin{tabular}{|l|l|l|l|}
\hline No. & Field Name & Field Type & Length \\
\hline 1 & Model type & VARCHAR2 & 40 \\
\hline 2 & Model name & VARCHAR2 & 50 \\
\hline 3 & Model files & VARCHAR2 & 250 \\
\hline 4 & Texture files & VARCHAR2 & 250 \\
\hline 5 & Model image & BLOB & --- \\
\hline 6 & Texture image & BLOB & --- \\
\hline 7 & Model properties & VARCHAR2 & 200 \\
\hline
\end{tabular}

(2) Realization of database management

OCI (Oracle Call Interface) was composed of file and library functions which provided by Oracle Corporation and as an application programming interface to access oracle database; it provides rich and efficient functions to manipulate BLOB data type. The realization of writing and reading model and texture files were through OCI functions. The warehouse management main interface shown in Figure 9 and model storage management shown in Figure 10.

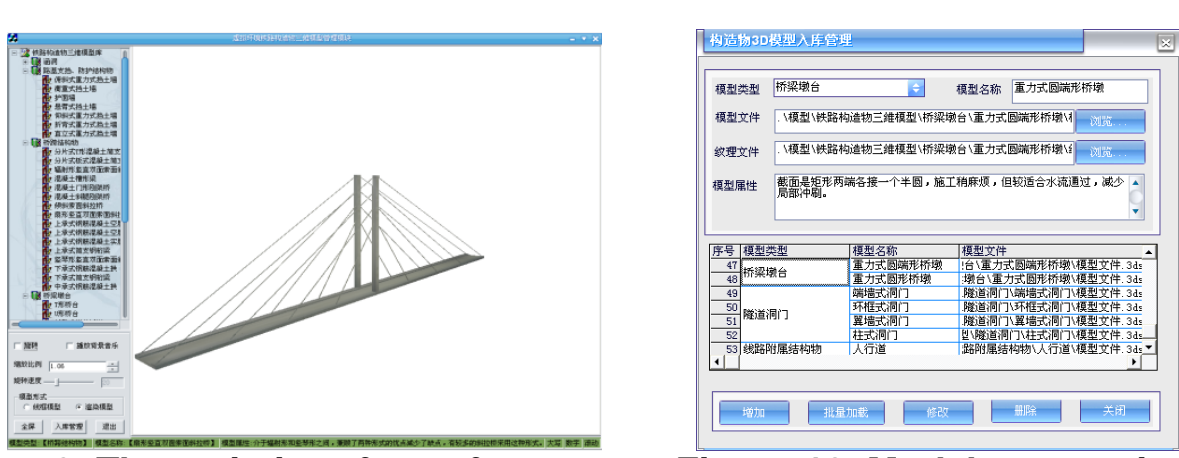

Figure 9. The main intefface of management

Figure 10. Model storage interface

\subsection{Three-dimensional modeling of line ancillary facilities}

The railway ancillary facilities including Catenary, line signs, etc. They have the same modeling method, three-dimensional modeling of catenary as an example to illustrate in here. In order to manage Catenary three-dimensional model effectively, established catenary model library and realized all catenary models add, modify and delete management. All models are stored in the Oracle database. Figure 11 was a catenary model storage mode interface; Figure 12 was a catenary model parameter setting interface.

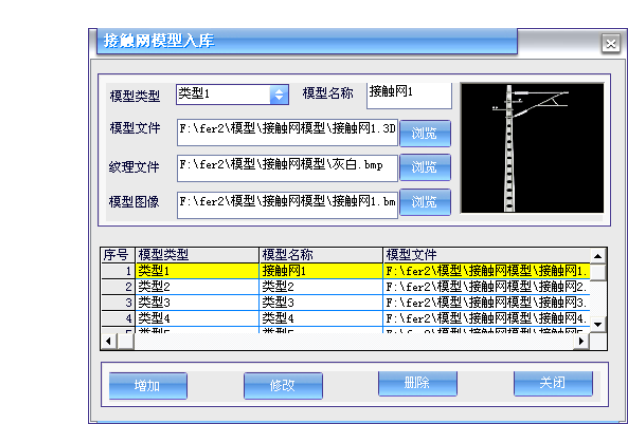

Figure 11. Catenary model storage interface

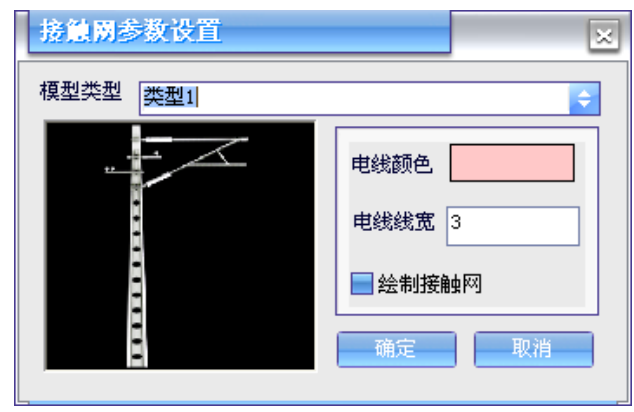

Figure 12. Model parameter settings 
Through calculated the three-dimensional coordinates of catenary model and read selected catenary 3D model, then rotating, zooming and panning operations, the model will be drawn in the calculated position. The catenary landscape modeling results shown in Figure 13.
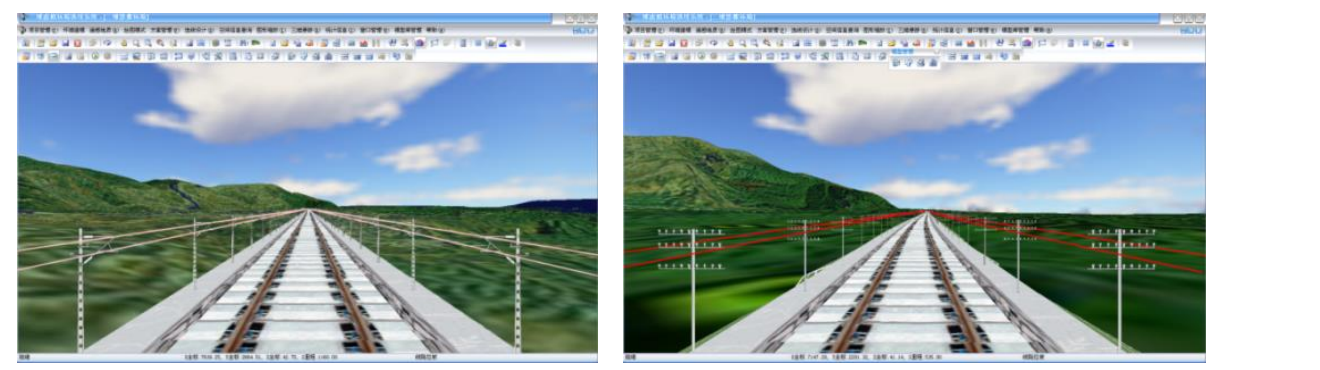

Figure 13. The catenary 3D landscape modeling renderings

\subsection{Three-dimensional model application in 3D environment}

The establishment of three-dimensional model was prerequisite, Managenent the threedimensional model was assurance, and the application of thee-dimensional model in threedimensional scene was the ultimate goal. The following vill diseuss the application of threedimensional model structures in the 3D environment.

1. The matching between three-dimensional nodel and three-dimensional scene

In the three-dimensional modeling study currently focused on a single model read and display problems, it not related to the specific three-dimensional scene environment. The three-dimensional application in railway line 3D enviromment must be solved matching with other three-dimensional models (sueh as three-dimensional lines model, etc.). There were three main issues must be addressed.

(1) Model position matching

Different types of structures determined the three-dimensional position in $3 \mathrm{D}$ environments. Such as piers should be in the bottom of the bridge deck. Only model position exact matching was correct and also 3D landscape required should be a whole.

(2) Model size matching

In establishing unit model, the nitial unit model size was not the same as corresponding size used in three-dimensional scene. Therefore, it need control the model size in threedimensional scene in order to achieve matching requirements.

(3) Model direction matching

This involved the problem of model rotation. It can achieve an exact model match position only three-dimensional model and associated model have the same direction.

2. Realization of model matching with 3D scene

In order to ensure an exact match, it must consider model transformation order questions, Namely, the order of rotation, zoom and pan transformation. OpenGL storage the model matrix transformation was based on a stack, According to the principle of the stack, LIFO, the efore, the following transformation sequence:

(1) Rotating operation, achieved model direction matching;

(2) Scaling operation, achieved model size matching;

(3) Panning operation, achieved model position matching.

The pier model as an example to illustrate, the core program as follows: void Private DrawBridgepier (double $\mathrm{x} 1$, double y1, double x2, double y2)

\{ 
m_maxX,m_minX: Maximum and minimum $\mathrm{x}$ coordinate value of model

m_maxY,m_minY: Maximum and minimum y coordinates value of model

// Get the deck width in three-dimensional scene to determine the pier model width float Xwidth $=\mathrm{m} \_$Railwaywidth $+2 * \mathrm{~m} \_$Ljwidth $+2 *$ m_GuiMianToLujianWidth; float mangle $=$ GetAngle $(\mathrm{x} 1, \mathrm{z} 1, \mathrm{x} 2, \mathrm{z} 2) ; \quad / /$ Calculate the piers rotation angle piers float $\mathrm{xscale}=\mathrm{X}$ width/(m_maxX $\left.-\mathrm{m} \_\operatorname{minX}\right) ; \quad / / \mathrm{X}$-axis direction scaling float yscale $=\mathrm{QDheight} /\left(\mathrm{m} \_\mathrm{max} Y-\mathrm{m} \_\min \mathrm{Y}\right) ; \quad / / Z$-axis direction scaling glPushMatrix (); glRotatef(mangle, $0,1,0)$; glScalef(xscale,yscale, 1$)$; // model rotation glTranslatef $\left(\left((x 1+x 2) / 2-\left(m \_m a x X+m \_m i n X\right) / 2\right) / x s c a l e\right.$, y2/yscale- m_maxY,(z1+z2)/2);// model translation glCallList(m_QDList);// Call the display list, showing the 3D model in 3D scenes glPopMatrix () ;

\}

Modeling results were shown in Figure 14.
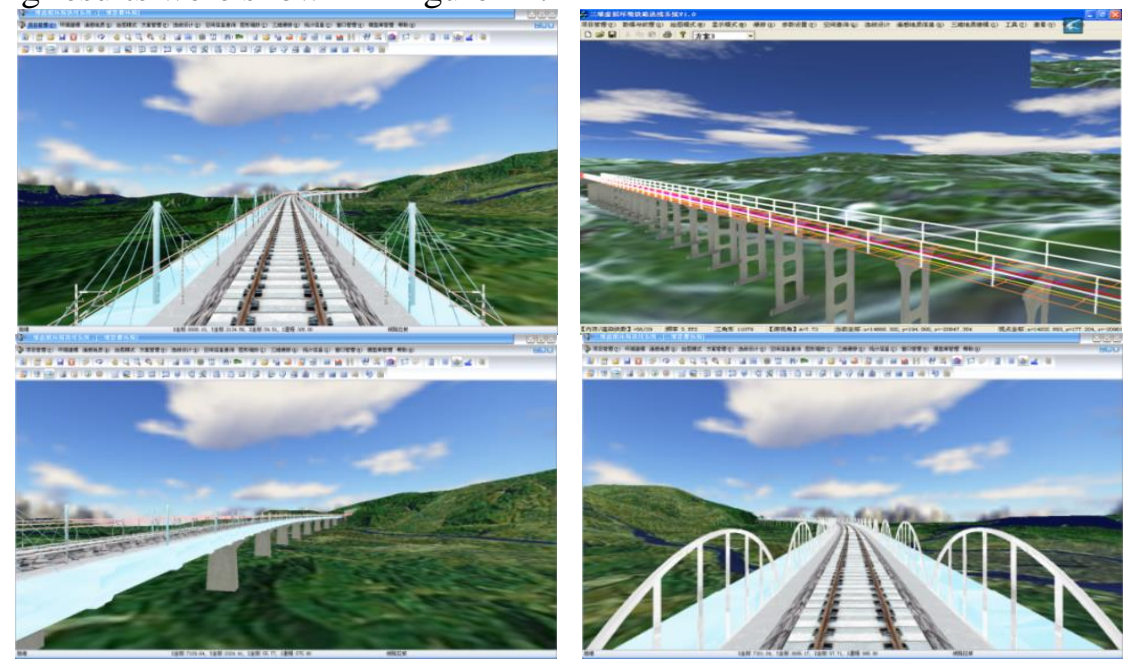

Figure 14. Three-dimensional modeling results of structures

\section{Trees modeling in line 3D landscape}

Trees were more typical plants of natural features, Generated realistic strong trees with different structurah eatures can greatly improve the authenticity of the scene.

\subsection{Three-dimensional modeling of trees based on graphics technology}

There has been significant progress in three-dimensional modeling of trees currently, mainly fractal theory, particle systems, geometric design method, feature integrated reasoning method and image-based modeling method [7-10]. The 3D trees realized by computer graphics had the shortcomings of large volumes data generally due to the trees' complex structure, It affect the overall efficiency of the software realization greatly in large threedimensional scene roaming. Studied the trees visual simulation from the perspective of digital image had advantage of strong realistic and easy interactive control. The image rendering technique commonly used was bill boarding technology, it used simple geometric texture mapping method replaced complex geometric drawings. Only sacrificed a little model authenticity, but reduced the difficulty of modeling and rendering time massively, improved 
the speed and efficiency and had little effect in real-time 3D rendering, Therefore, the paper adopt this modeling method to realize the trees modeling in line 3D landscape .

\subsection{Acquisition and processing of data resources}

The main work of modeling tree was rotated the image as much as possible to make the tree trunk perpendicular direction, used image processing software such as Photoshop dealt the trees surrounding color pure black or pure white. The before and after treatment effect was shown in Figure 15. The system provides nearly 30 species of trees, as shown in Figure 16.
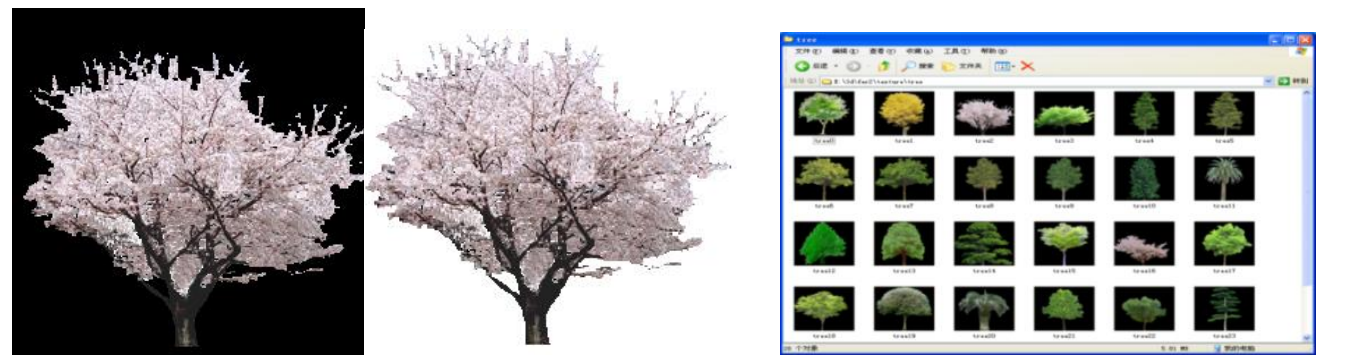

Figure 15. The effect of before and after treatment

\subsection{Realization of trees three-dimensional modeling}

Figure 16. The tress model library

Load the image into system and expanded three-colon mage (RGB) into four-color (RGBA), when met judge the background color, distrbuted the alpha value transparent completely in order to create a non-rectangular raster mages. Through uses texture mapping, alpha blending techniques and axiat Bill boarding technology, Make the two-dimensional image of trees cross affixed specific location an achieved realistic effects. Parameter settings shown in Figure 17, Final modeling results shown in Figure 18.

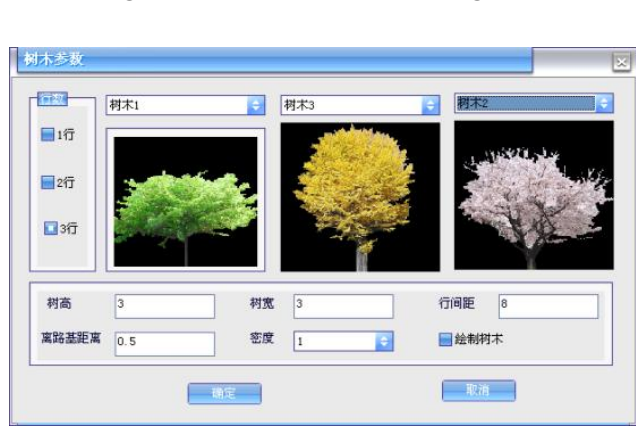

Figure 17. 3D trees parameters settings

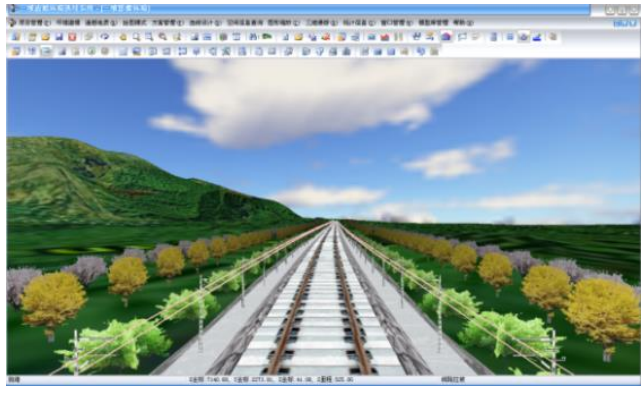

Figure 18. 3D trees rendering effect

\section{Conclusions}

The paper researched and discusses the key technologies and methods to build threedimensional landscape lines involved. Experimental results shown that used bi-cubic coons surface model, Object-oriented graphics assisted simulation and unit-level modeling approach, Construction of the line model library for managing 3D landscape model can assistant engineer decision-making landscape model selection in three-dimensional visual design, Improved the efficiency of three-dimensional real-time design, created a 3D landscape with a higher fidelity line became possible. The image-based methods was more suitable for trees modeling in line 3D landscape environment, It can be display three-dimensional landscape of 
trees flexibly and realistically, improved authenticity and roaming rate of line landscape. For OpenGL graphics processing was very powerful, but lack the ability to complex physical modeling features, used the third-party modeling tools to build the railway line structure models. Solved the problem of exact match between three-dimensional model and the overall three-dimensional scene, achieved the application of railway structure models in virtual threedimensional scene, and built a unified landscape of three-dimensional line selection environment.

\section{Acknowledgements}

This research was supported by the National Natural Science Foundation of China (Project No.: 51278316); the natural science foundation of Hebei Province, China (Project No.: E2014210111); The Key Project of Education Department of Hebei Province, China (Project No.: ZD20131026).

\section{References}

[1] P. Hao, "Study on Key Technologies in Developing Modern RoadCAD System" Central South University doctoral dissertation (2002).

[2] Z. Yi-fei, Y. Shao-wei, X. Jin-liang, "Vertical design method of ntersection based on bilinear Coons surface model", Journal of Traffic and Transportation Engineering, vol. 3, (2003), pp.17-20.

[3] C. Zhiming, Y. Jun, and Z. Yingjie, "Multi-Agent/Multi-Goal Modeling Templates and the 1-1, n-1,1-n, n-n Deductions of Relationship-chain [J]", Internationa1 Journal of Hybrid Information Technology, vol. 3, no.1, (2010) January, pp. 49-64.

[4] L. Yan and H. Xu-li, "Mixed Coons patch with shape parameters", Computer Engineering and Applications [J]", vol. 45, no. 8, (2009), pp. 72-74.

[5] K. L. Shi, J. H. Yong, J. G. Sun and J. C.Paul, "Gn blending multiple surfaces in polar coordinates", Computer Aided Design, vol. 42, (2010),pp. 479-494.

[6] W. Yuhua and Y. Kejiang, "Application of Object oriented to 3D Scenery Modeling. Journal of Wuhan University of Technology”, Transportation Science and Engineering, vol. 27, (2003), pp. 37-40.

[7] J. Xiaojin and M. Yaohai, "Realistic simulation of three-dimensional trees [J]",Computer Engineering and Design, vol. 31, (2010), Pp 1753-1756.

[8] D. Huan, W. Wanggen, X Xiaoping, and T. Xiaohui, "Realistic simulation of plant based on geometry parameter", Journal of Computer Applications, vol. 29, (2009), pp. 97-100.

[9] L. Ding, C. Chong-cheng, T. Li-yu and Z. Jie, "3D tree modeling based on the parametric curve and its integral [J]", Journal of Fuzhoutuniversity (Natural Science Edition), vol. 39, (2011), pp. 367-374.

[10] A. Rechemartinez, I. Martin and Drettakis, "Volumetric reconstruction and interactive rendering of trees from photographs [J]”, ACM Trans Graph, vol. 23, (2004), pp. 720 -727.

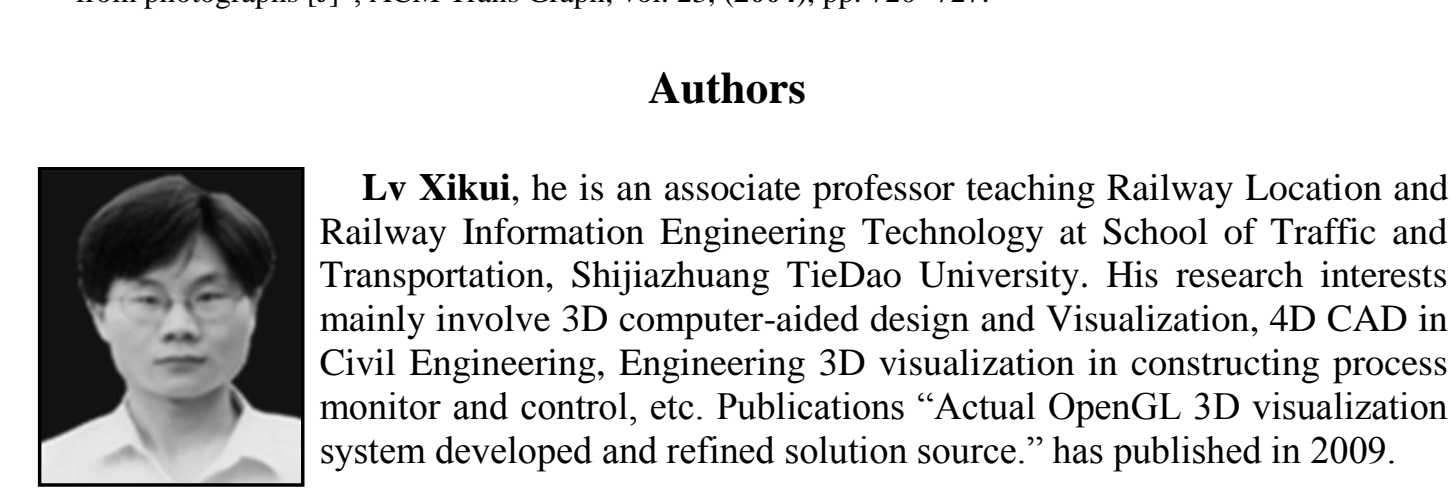




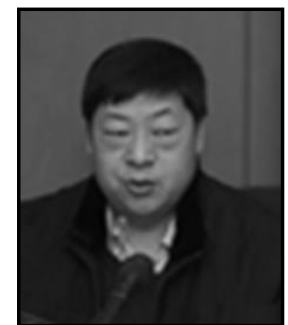

Wang Mingsheng, he is a professor teaching Urban Rail Transit Planning and Design and Geographic Information Systems at School of Civil Engineering, Shijiazhuang TieDao University. His research interests mainly involve Railway Survey and Design Integration of information technology, GIS technology and its applications and traffic information technology, etc. Publications "Urban Rail Transit Planning and Design." and "Urban Rail Transit System Introduction" has published in 2011.

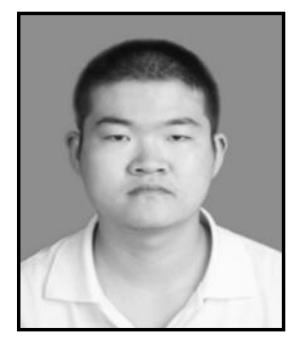

He Bin, he was bored in 20 June, 1989, He is a graduate and studying at the School of Civil Engineering of Shijiazhuang TieDao University now, His research interests mainly involve the three-dimensional visualization engineering design, the three-dimensional GIS, etc.

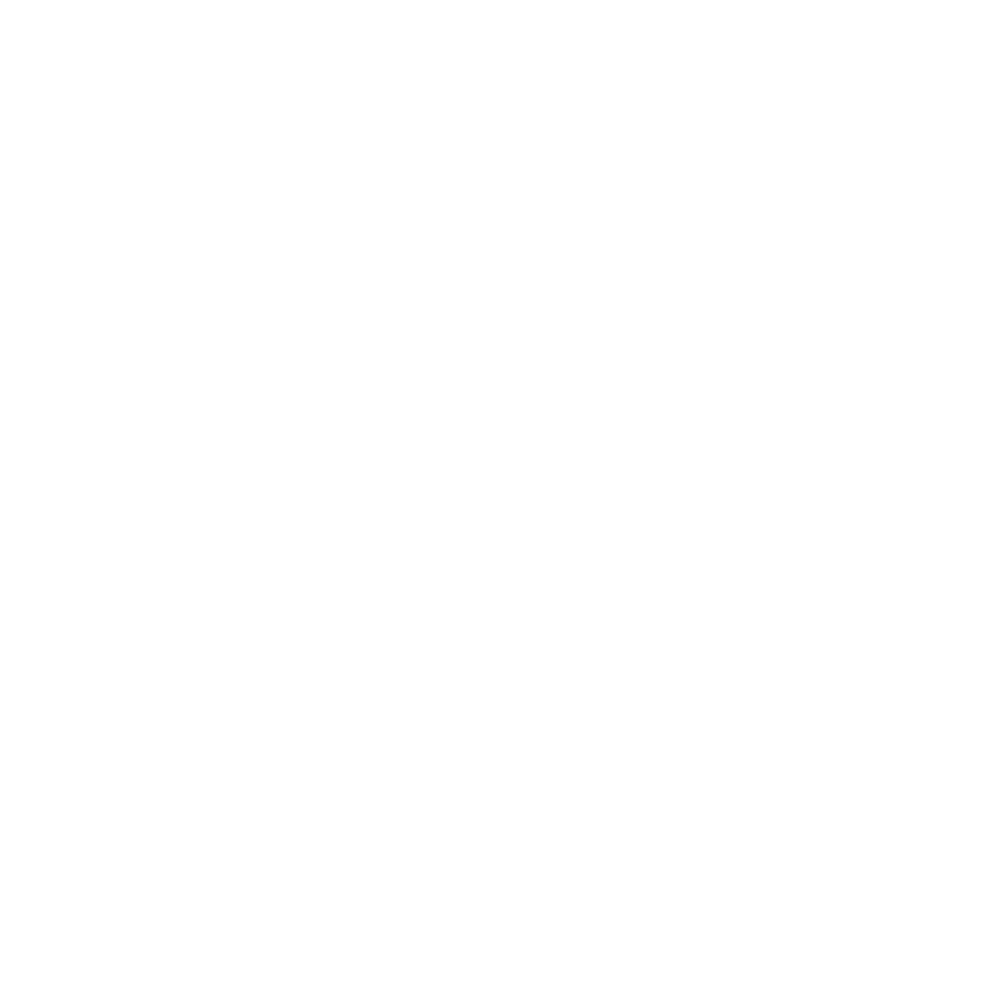


International Journal of Hybrid Information Technology

Vol.7, No.3 (2014)

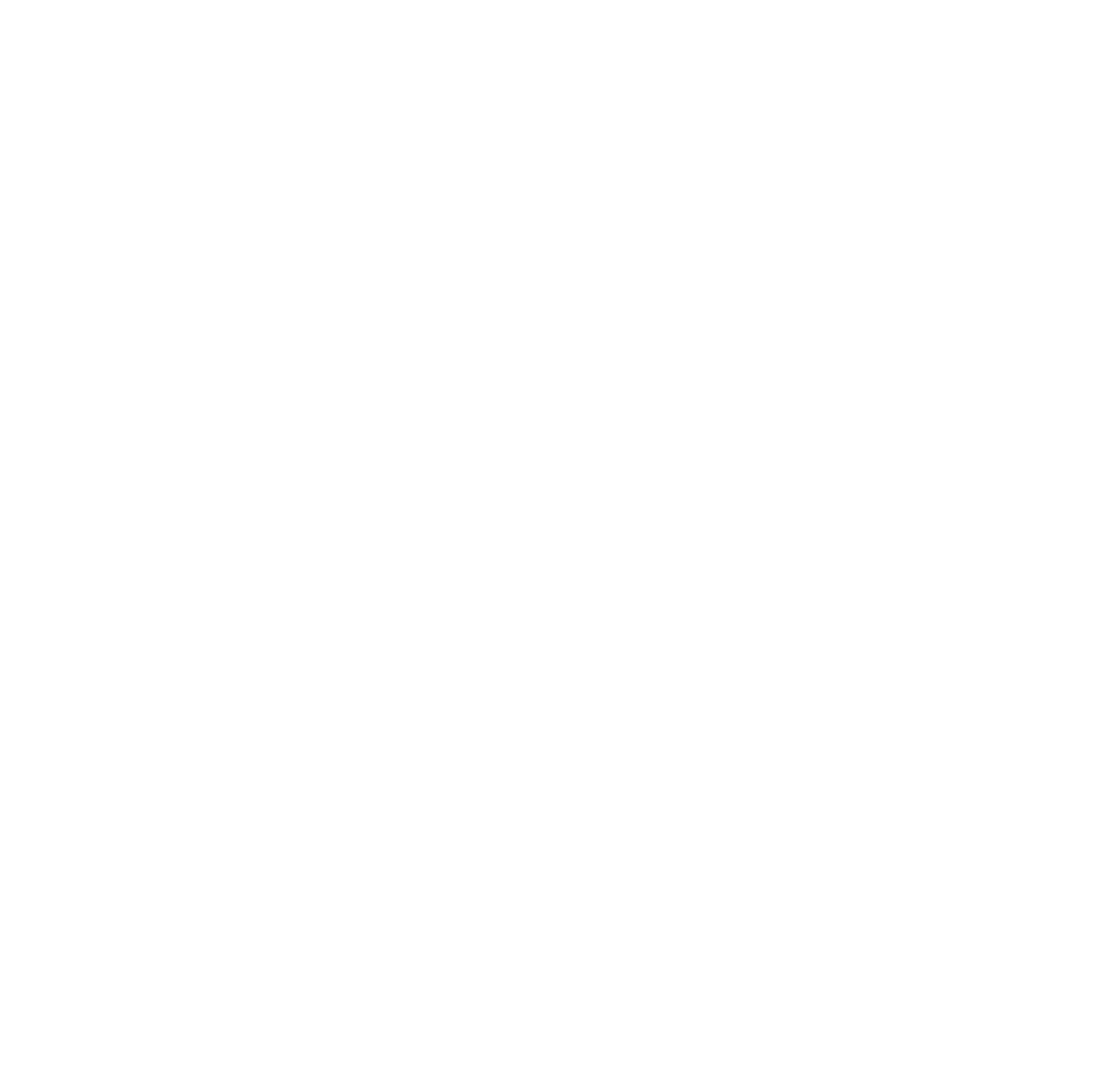

